\title{
ESTUDO DO PROCESSO DE SECAGEM CONVECTIVA DO PIMENTÃO (Capsicum annuum L.)
}

Study of the convective drying process of pepper (Capsicum annuum L.)

Estudio del processo de secado convectivo del pimiento (Capsicum annuum L.)

\section{Desafios}

Artigo Original Original Article Artículo Original

Edwallas Lima Sousa ${ }^{1}$, Djany Souza Silva ${ }^{1}$, Antonia Mayara Brilhante de Sousa $^{1}$, Renata de Araújo Alves ${ }^{1}$, Virgínia Kelly Gonçalves Abreu ${ }^{1}$, Ana Lúcia Fernandes Pereira $^{* 1}$

${ }^{1}$ Laboratório de Química de Alimentos, Curso de Engenharia de Alimentos, Universidade Federal do Maranhão, Imperatriz, Brasil.

*Correspondência: Centro de Ciências Sociais, Saúde e Tecnologia, Universidade Federal do Maranhão, Av. da Universidade, S/N, Dom Afonso Felipe Gregory, Imperatriz, Maranhão, Brasil. CEP:65.915-240. e-mail anafernandesp@hgmail.com.

Artigo recebido em 24/02/2020 aprovado em 23/03/2020 publicado em 26/03/2020.

\section{RESUMO}

O objetivo deste trabalho foi estudar a cinética de secagem do pimentão (Capsicum annuum L.) em estufa com circulação forçada de ar a $80^{\circ} \mathrm{C}$, através de diferentes modelos matemáticos. Para isso, foram utizados pimentões, os quais foram fatiados nas dimensões $3,0 \mathrm{~cm}$ de comprimento, $2,5 \mathrm{~cm}$ de largura e $0,5 \mathrm{~cm}$ de espessura. As fatias foram dispostas em bandejas perfuradas de alumínio. As equações de Lewis, Page, Logarítmica e de Henderson e Pabis foram ajustadas para os dados experimentais e o grau de ajuste foi avaliado mediante o coeficiente de determinação $\left(\mathrm{R}^{2}\right)$ e erro médio relativo. De acordo com os resultados, observou-se o tempo de secagem de 420 min para peso estável na temperatura de $80^{\circ} \mathrm{C}$ e atividade de água (Aw) de 0,676. A manutenção de um baixo valor de Aw é essencial para garantir a estabilidade microbiológica do produto. O modelo de Lewis, para a faixa de tempo de secagem entre 0 a 180 min foi o que apresentou melhor ajuste matemático à curva experimental, obtendo o maior $\mathrm{R}^{2}$ e menor valor de P. Desta forma, esse modelo apresenta-se com o mais recomendado para a predição do comportamento da secagem do pimentão à $80^{\circ} \mathrm{C}$.

Palavras-chave: Estabilidade microbiológica, equações matemáticas, modelagem.

\section{ABSTRACT}

The aim of this study was to evaluate the drying kinetics of pepper (Capsicum annuum L.) in a greenhouse with forced air circulation at $80^{\circ} \mathrm{C}$, using different mathematical models. For this, it was used peppers, which were sliced in the dimensions $3.0 \mathrm{~cm}$ in length, $2.5 \mathrm{~cm}$ wide and $0.5 \mathrm{~cm}$ thick. The sliced were dried in perforated aluminum trays. The Lewis, Page, Logarithmic, and Henderson and Pabis equations were adjusted for the experimental data, and the degree of fit was evaluated by the coefficient of determination $\left(R^{2}\right)$ and relative mean error. According to results, the drying time was 420 min for stable weight at $80^{\circ} \mathrm{C}$ and water activity (Aw) of 0.676. Maintaining a low Aw value is essential to ensure the microbiological stability of the product. The Lewis model, for the drying time range from 0 to $180 \mathrm{~min}$, had the best mathematical fit to the experimental curve, obtaining the highest $R^{2}$ and the lowest $P$ value. Thus, this model presents the more recommended for predicting the drying behavior of peppers at $80^{\circ} \mathrm{C}$.

Keywords: Microbiological stability, mathematical equations, modelling.

\section{RESUMEN}

El objetivo de este trabajo fue estudiar la cinética de secado de pimientos (Capsicum annuum L.) en un horno con circulación forzada de aire a $80^{\circ} \mathrm{C}$, utilizando diferentes modelos matemáticos. Para esto, se usaron pimientos, que se cortaron en rodajas en dimensiones de $3.0 \mathrm{~cm}$ de longitud, $2.5 \mathrm{~cm}$ de ancho y $0.5 \mathrm{~cm}$ de espesor. Las rodajas 
fueron colocadas en bandejas de aluminio perforado. Las ecuaciones de Lewis, Page, Logarithmic y Henderson y Pabis se ajustaron para los datos experimentales y el grado de ajuste se evaluó utilizando el coeficiente de determinación $\left(R^{2}\right)$ y el error promedio relativo. De acuerdo con los resultados, se observó un tiempo de secado de 420 min para un peso estable a una temperatura de $80^{\circ} \mathrm{C}$ y una actividad de agua (Aw) de 0,676. Mantener un valor Aw bajo es esencial para garantizar la estabilidad microbiológica del producto. El modelo de Lewis, para el rango de tiempo de secado entre 0 y 180 min, fue el que mejor ajuste matemático a la curva experimental, obteniendo el valor $R^{2}$ más alto y el valor P más bajo. Por lo tanto, este modelo se presenta con el más recomendado para la predicción del comportamiento de secado del pimiento a $80^{\circ} \mathrm{C}$.

Descriptores: Estabilidad microbiológica, ecuaciones matemáticas, modelado.

\section{INTRODUÇÃO}

O pimentão (Capsicum annuum L.) pertence à família das solanáceas, assim como a batata, o tomate, a berinjela e as pimentas em geral. Segundo Butruille (2018), o pimentão é uma das dez hortaliças de maior importância econômica.

Juntamente com outras espécies do gênero Capsicum, o pimentão é cultivado mundialmente, e segundo FAOSAT (2016), a China tem a produção de 17,5 milhões de toneladas/ano, correspondendo a mais da metade da produção mundial. O país tem maior destaque no cenário internacional, seguida de México, Turquia e Indonésia com 2,7, 2,5 e 2 milhões de toneladas/ano, respectivamente.

No Brasil, o cultivo do pimentão ocupa uma área equivalente a 13 mil hectares (ha). A produção é de 350 mil toneladas (t) de frutos. No âmbito nacional, os principais produtores do fruto são os estados de São Paulo, Minas Gerais, Ceará, Distrito Federal, Rio de Janeiro, Espírito Santo e Pernambuco, que respondem à $87 \%$ do total produzido (sendo os dois primeiros responsáveis, sozinhos, por 40\%) (ABRAFRUTAS, 2019).

Embora o pimentão possa ser cultivado em campo aberto, apresenta resultados proeminentes em cultivo protegido devido ao melhor controle das condições ambientais e dos tratos culturais. Segundo Henz et al. (2007), o pimentão cultivado em campo tem produtividade entre 25 e 40 toneladas por hectares (t/ha), enquanto em cultivo protegido pode atingir a produção de 180 t/ha. Em áreas com períodos de chuvas intensas, como a região Norte e a Centro Oeste e em regiões com invernos frios, como a região Sul são exemplos de lugares onde este sistema de cultivo é adotado (RAGASSI; MELO, 2017).

O fruto é comumente consumido verde ou maduro, fresco ou industrializado. $\mathrm{Na}$ indústria de alimentos, são utilizados como conservas, condimentos, molhos e como matéria-prima para a extração de corantes alimentares e oleorresinas (MAROTO; SORIA, 2017).

$\mathrm{Na}$ comercialização dos frutos e hortaliças in natura, a qualidade é afetada pela alta perecibilidade, associada a ações inadequadas no pós-colheita que desfavorecem sua conservação. Isto porque a qualidade na pós-colheita é significativamente afetada pelo cultivar, estádio de maturação no momento da colheita, temperatura de armazenamento e qualidade inicial do produto, o que reduz o tempo de comercialização do pimentão (MORGADO et al., 2008). Assim, a secagem representa um processo alternativo na conservação do fruto. Nas últimas décadas, pesquisas sobre secagem têm sido largamente ampliadas em busca de produtos com poucas alterações em suas características sensoriais e nutritivas (SILVA et al., 2017).

Para Ferreira (2017), o processo de secagem de frutos melhora a estabilidade microbiológica, química e enzimática do produto, facilita o transporte e armazenamento, além de ofertar um produto 
diferenciado. O processo envolve, simultaneamente, a transferência de calor e de massa, em que grande parte da água presente no material poroso é removida por evaporação em um meio gasoso insaturado (DIAS, 2013; SILVA, 2015). Ao reduzir o teor de umidade de um produto, um dos principais fatores que determinam a elevada taxa de deterioração é eliminado ou minimizado (FERNANDES et al., 2015).

A secagem pode ser realizada em equipamentos como a estufa ou secadores tipo túnel, que são de simples construção e funcionamento (DIAS, 2013). A cinética de secagem, ou seja, a rapidez com que o alimento perde umidade é controlada pelas características da matriz do alimento e pelas variáveis: temperatura, velocidade e umidade relativa do ar e representada através das curvas de secagem (GRAÇA, 2015). A partir da análise das curvas de secagem, o dimensionamento, a otimização e a determinação da viabilidade de sua aplicação comercial, podem ser feitos por meio de simulações matemáticas, a qual utiliza modelos matemáticos que descrevem satisfatoriamente a perda de água durante o processo de secagem. Atualmente, a literatura apresenta diferentes modelos matemáticos capazes de descrever a secagem de produtos agrícolas ao longo do tempo, sendo eles, teóricos, semi-teóricos e os empíricos (ARAÚJO et al., 2017). Diante disso, o presente trabalho teve como objetivo estudar a cinética de secagem convectiva do pimentão a $80^{\circ} \mathrm{C}$, por meio dos modelos matemáticos semi-empíricos para a predição da razão da umidade ao longo do tempo de secagem.

\section{MATERIAIS E MÉTODOS}

O estudo foi realizado no laboratório de Química de Alimentos do curso de Engenharia de Alimentos da Universidade Federal do Maranhão. Utilizou-se pimentões (Capsicum annuиm L), adquiridos no comercio local da cidade de Imperatriz,
MA. Os frutos foram selecionados e higienizados em água clorada (100 ppm/15 min), e, em seguida, foram retiradas as sementes e fatiados. Obteve-se fatias de pimentão nas dimensões $3,0 \mathrm{~cm}$ de comprimento; 2,5 $\mathrm{cm}$ de largura e $0,5 \mathrm{~cm}$ de espessura, as quais foram dispostas em bandejas perfuradas de alumínio.

Para a secagem, utilizou-se estufa de circulação forçada de ar (MA35, Marconi, Piracicaba, Brasil), a temperatura de $80{ }^{\circ} \mathrm{C}$. A perda de peso foi monitorada ao longo do tempo através de balança analítica (AY220, SHIMADZU, Quioto, Japão) em intervalos regulares, em triplicata.

A atividade de água foi determinada através do equipamento AQUALAB digital (S4TE, DECAGON, São José dos Campos, Brasil).

O cálculo da razão da umidade (RU) durante a secagem foi realizado conforme a Equação 1. Onde U é a umidade do produto no tempo (b.s.); Uo é a umidade inicial do produto (b.s.); e, Ue é a umidade de equilíbrio (b.s.).

$$
R U=\frac{U-U_{e}}{U_{0}-U_{e}}
$$

Os dados obtidos para RU foram ajustados para os modelos matemáticos de Lewis, Page, Henderson, e, Logarítmico (Tab 1). O ajuste dos parâmetros matemáticos das equações foram obtidos a partir do algoritmo de minimização LevenbergMarquardt (método de mínimos quadrados não-linear) implementado no software OriginPro versão 9.0.

Tabela 1. Modelos matemáticos da literatura, avaliados para predizer razão da umidade do processo de secagem do pimentão.

\begin{tabular}{lll}
\multicolumn{1}{c}{ Modelo } & \multicolumn{1}{c}{ Equação* $^{*}$} & \multicolumn{1}{c}{ Referência } \\
\hline \multirow{2}{*}{ Lewis } & $R U=\exp (-k t)$ & $\begin{array}{l}\text { (AKPINAR; } \\
\text { BICER; } \\
\text { CETINKAYA, } \\
\text { 2006) }\end{array}$ \\
Page & $R U=\exp \left(-k t^{n}\right)$ & (LEWIS, 1921) \\
Logarítmico & $R U=a \exp (-k t)+c$ & (SANTOS et al., \\
& &
\end{tabular}


Henderson

$$
R U=a \exp (-k t)
$$

(DIAMANTE; MUNRO, 1993)

*a, k, n e c são constantes empíricas dos modelos (adimensionais). t é o tempo em minutos.

$$
P=\frac{100}{n} \sum_{i=1}^{n} \frac{\left|R U_{\text {exp }}-R U_{\text {calc }}\right|}{R U_{\text {exp }}}
$$

Onde n é o grau de liberdade, $\mathrm{RU}_{\text {exp }}$ é a razão da umidade experimental e $\mathrm{RU}_{\text {calc }}$ é a razão da umidade calculada.

Utilizou-se o coeficiente de determinação $\left(\mathrm{R}^{2}\right)$ e o erro médio relativo $(\mathrm{P})$ para escolher o modelo matemático que melhor descreveu a curva da cinética da secagem do pimentão.

\section{RESULTADOS E DISCUSSÃO}

Na Figura 1 é apresentada a curva de atividade de água (Aw) ao longo do tempo de secagem do pimentão. É possível observar que ao longo do tempo houve uma redução da Aw do pimentão. Isto advém das condições proporcionadas pelo aquecimento da amostra, tornando possível a transferência simultânea de calor e de massa, principalmente, na superfície do material, promovendo a remoção de água do mesmo (FERNANDES, 2018).

Segundo Fellows (2018) e Garcia (2014), praticamente toda a atividade microbiana é inibida quando a atividade de água (Aw) encontra-se abaixo de 0,6. Assim, observa-se que o pimentão não apresentou Aw abaixo desse valor. Ou seja, dentro do estabelecido por estes autores a estabilidade microbiológica do produto poderá ser prejudicada, por obter valor de 0,67 para Aw após 420 min de secagem. Sendo assim, é recomendado combinar com outros mecanismos de conservação pimentão.

Entretanto, de acordo com Jay (2005), alimentos desidratados possuem a faixa de Aw de 0,60 a 0,75 para manter a estabilidade microbiológica. Diante disto, constata-se que o pimentão desidratado neste trabalho apresenta-se dentro da faixa de atividade de água que poderá possibilitar a inibição microbiana a longo prazo, desde que acondicionado adequadamente. Esse dados corroboram com àqueles obtidos por Sousa et al. (2019) que também alcançou valores semelhantes.

Figura 1. Atividade de água do pimentão ao longo do tempo de secagem convectiva a $80{ }^{\circ} \mathrm{C}$.

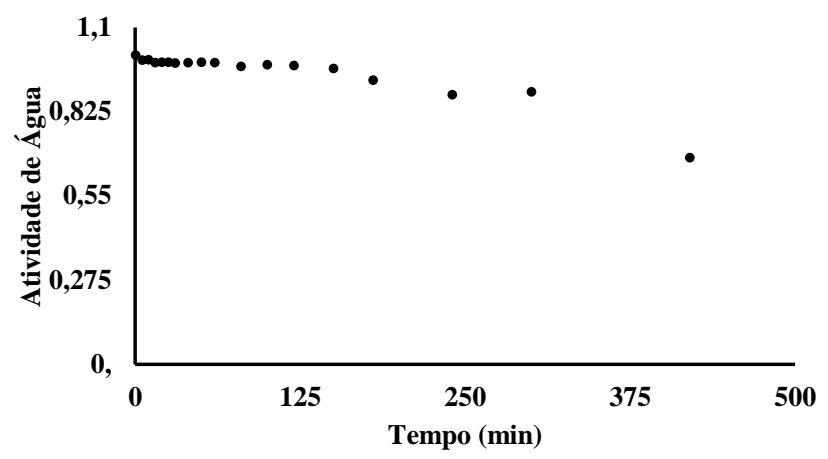

Na Figura 2, observa-se a curva da razão da umidade durante a secagem do pimentão obtida experimentalmente à temperatura de $80^{\circ} \mathrm{C}$ e os ajustes dos modelos matemáticos aplicados. Esses resultados estão de acordo com àqueles reportados por Tsuda et al. (2014) e Faria et al. (2012), onde o ajuste das curvas preditas para os 4 modelos avaliados se mostraram praticamente o mesmo, com boa aproximação dos dados experimentais.

Figura 2. Curvas de secagem do pimentão com ajuste dos dados experimentais pelos modelos matemáticos de Lewis, Henderson, Logarítmico e Page.

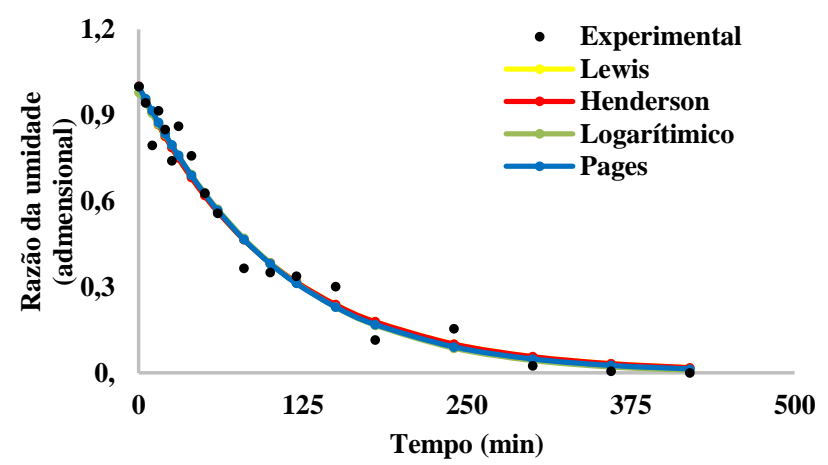

Os parâmetros empíricos dos modelos ajustados são apresentados na Tabela 2 com os valores do coeficiente de correlação $\left(\mathrm{R}^{2}\right)$ e erro médio relativo 
(P). De acordo com Madamba et al. (1996), o coeficiente de determinação $\left(\mathrm{R}^{2}\right)$, não constitui, sozinho, um bom critério para a seleção de modelos não lineares. Para isto, os valores para o erro médio relativo (P) também foram considerados. Uma vez que, em acordo com Akpinar (2006), Tsuda et al.
(2014) e Moraes Filho et al. (2014), quanto maior for o coeficiente de determinação e menor o erro médio relativo, o modelo é considerado adequado pois calcula os parâmetros mais próximos possível dos dados experimentais.

Tabela 2 - Parâmetros, coeficientes de determinação $\left(\mathrm{R}^{2}\right)$ e erro médio relativo $(\mathrm{P})$ dos modelos matemáticos de Lewis, Henderson \& Pabis, Logarítmico e Page.

\begin{tabular}{|c|c|c|c|c|c|c|c|}
\hline \multirow[t]{2}{*}{ Equação } & \multicolumn{4}{|c|}{ Parâmetros } & \multirow[t]{2}{*}{$P(0$ - $420 \mathrm{~min})$} & \multirow[t]{2}{*}{$P(0$ - $180 \mathrm{~min})$} & \multirow[t]{2}{*}{$\mathbf{R}^{2}$} \\
\hline & $\mathbf{a}$ & K & $\mathrm{c}$ & $\mathrm{N}$ & & & \\
\hline Lewis & - & 0,01742 & - & - & 89943,91397 & 10,99387 & 0,97216 \\
\hline Henderson & 1,14000 & 0,02470 & - & - & 89311,99885 & 11,00220 & 0,97216 \\
\hline Logarítimico & 1,00590 & 0,01706 & $-2,5 \mathrm{E}-05$ & 1,03220 & 43164,77045 & 11,20307 & 0,97300 \\
\hline Pages & - & 0.01720 & - & 1.02980 & 67845,91443 & 11.00218 & 0,97260 \\
\hline
\end{tabular}

Analisando os dados da Tabela 2, em relação aos coeficientes de determinação $\left(\mathrm{R}^{2}\right)$, observa-se valores próximos de 1 para todos os modelos. Segundo Madamba et al. (1996) e Martinazzo et al. (2007), os valores de $\mathrm{R}^{2}$ quanto mais próximos de 1 , denotam um excelente ajuste dos modelos para representação da cinética de secagem. Silva et al. (2015) obtiveram resultado semelhante para a secagem dos frutos do açaí.

No entanto, uma vez que o $\mathrm{R}^{2}$ não é capaz de, sozinho, determinar satisfatoriamente o melhor modelo, torna-se viável a avaliação dos erros médios relativos $(\mathrm{P})$. Em relação a esses valores, é possível notar que para o tempo de secagem até $420 \mathrm{~min}$, nenhum dos modelos obteve valor de $\mathrm{P}$ aceitável, muito embora apresente bom coeficiente de correlação $\mathrm{R}^{2}$. Assim, os modelos foram remodelados para a faixa de tempo entre 0 e 180 min, e percebe-se que há uma enorme discrepância entre a faixa inicial de 0 a 420 min de secagem. Isto indica que os quatro modelos são ineficientes para descrever o comportamento da secagem após tempo de $180 \mathrm{~min}$, visto que o erro médio encontrado para o tempo de 0 a $420 \mathrm{~min}$ apresentaram valores elevados.

A necessidade de recalcular o erro tornou-se importante pois após $180 \mathrm{~min}$ de secagem os valores de $\mathrm{P}$ encontrados foram altos, em discordância com a literatura (ARAÚJO et al., 2017; MOSCON et al., 2017). Enquanto para tempo de secagem até $180 \mathrm{~min}$, foram obtidos erros médios baixos, sendo considerado um critério importante na escolha dos modelos, ou seja, acima de $180 \mathrm{~min}$ de secagem, as equações matemáticas avaliadas neste trabalho não possuem boa predição da curva de secagem do pimentão dentro das condições avaliadas neste trabalho.

Assim, para a faixa reduzida de 0 até $180 \mathrm{~min}$, o modelo que mais se adequou ao comportamento da secagem foi o modelo de Lewis, pois o mesmo apresentou um valor de $\mathrm{P}$ menor $(10,993879 \%)$ e $\mathrm{R}^{2}$ considerável $(0,9722)$. Este resultado corrobora com o de Moraes Filho et al. (2014), que avaliaram a aplicação de modelos empíricos da secagem 
convectiva da acerola. De acordo com esses autores, os resultados obtidos demonstraram que os modelos Lewis descreveram satisfatoriamente 0 comportamento cinético do processo de secagem para as condições de operação trabalhadas.

Observa-se ainda na Tabela 2 que, o modelo Pages apresenta valor de $\mathrm{P}$ próximo do valor obtido para o modelo de Lewis. Ambos os modelos podem ser aplicados na descrição matemática da secagem do pimentão até 180 min de secagem.

\section{CONCLUSÃO}

Diante dos resultados, foi possível observar que na condição de secagem avaliada, o tempo de secagem do pimentão de $420 \mathrm{~min}$, permitiu obter valores de atividade de água que podem garantir a estabilidade do produto. A predição matemática da secagem do fruto a $80{ }^{\circ} \mathrm{C}$ em estufa com circulação forçada de ar foi melhor quando aplicado a equação matemática de Lewis, a qual apresentou-se como a mais indicada, dentro da faixa para predizer a cinética de secagem na condição estudada.

\section{AGRADECIMENTO}

Agradecimentos a Conselho Nacional de Desenvolvimento Científico e Tecnológico (CNPq) no âmbito do INCT - Frutos Tropicais pelo financiamento e Bolsa de Iniciação Científica. Ao Programa Institucional de Bolsas de Iniciação Científica - PIBIC, a Universidade Federal do Maranhão - UFMA e ao Grupo de Pesquisa Ciência e Tecnologia de Alimentos.

Todos os autores declararam não haver qualquer potencial conflito de interesses referente a este artigo.

\section{REFERÊNCIAS}

ABRAFRUTAS, Associação Brasileira dos Produtores Exportadores de Frutas e Derivados. Cenário Hortifruti Brasil, 2018. Disponível em: $<$ https://abrafrutas.org/2018/10/31/relatorio-cenario- hortifruti-brasil-2018-mostra-que-geracao-deempregos-e-destaque/>. Acesso em: 10 out, 2019.

AGRICULTURA, Confederação Nacional da. Mapeamento e quantificação da cadeia produtiva das hortaliças do Brasil. 79 p. Brasília, DF, 2017.

AKPINAR, E. K.; BICER, Y.; CETINKAYA, F. Modelling of thin layer drying of parsley leaves in a convective dryer and under open sun. Journal of Food Engineering, v. 75, n. 3, p. 308-315, 2006.

ARAÚJO, C. D. S.; MACEDO, L. L.; VIMERCATI, W. C., SARAIVA, S. H.; OLIVEIRA, A. D. N.; TEIXEIRA, L. J. Q. Foam-mat drying kinetics for acerola and adjustment of the mathematical models. Brazilian Journal of Food Technology, v. 20, 2017.

ARAUJO, W. D.; GONELI, A. L. D.; CORRÊA, P. C.; HARTMANN FILHO, C. P.; MARTINS, E. A. S. Modelagem matemática da secagem dos frutos de amendoim em camada delgada. Revista Ciência Agronômica, v. 48, n. 3, p. 448-457, 2017.

BUTRUILLE, N. S. Utilização de hidrogel nanocompósito com liberação controlada de nureia em substrato para produção de mudas de pimentão (Capsicum annuum L.). Universidade de Brasília / Faculdade de Agronomia e Medicina Veterinária, 2018.

DIAMANTE, L. M.; MUNRO, P. A. Mathematical modelling of the thin layer solar drying of sweet potato slices. Solar Energy, v. 51, n. 4, p. 271-276, 1993.

DIAS, L. G. Estudo do processo de secagem em estufa e por microondas de compósitos cerâmicos de argila e resíduos de esteatito. 2013. Dissertação (mestrado em Engenharia da Energia) - Departamento de Engenharia Mecânica. Universidade Federal de São João del Rei. Departamento de Ciências Térmicas e dos Fluidos e Centro Federal de Educação Tecnológica de Minas Gerais, 2013.

FAO. FAOSTAT. Production quantities of Chillies and peppers, green by country. 2016. Disponível em:<http://www.fao.org/faostat/en/\#data/QC/visualiz e> Acesso em: 10 out. 2019.

FARIA, R. Q. De; TEIXEIRA, I. R.; DEVILLA, I. A.; ASCHERI, D. P.; RESENDE, O. Cinética de secagem de sementes de crambe. Revista Brasileira de Engenharia Agrícola e Ambiental, v. 16, n. 5, p. 573-584, 2012.

FERNANDES, A. I.; DE LÓCIO, C. J. F.; DE MORAIS, M. S. N.; BRUNO R. P. N. Análise da Cinética de Secagem de Mallus Domestica em 
Estufa. Congresso Técnico Científico da Engenharia e da Agronomia CONTECC. Maceió - Alagoas, 2018.

FELLOWS, P. J. Tecnologia do Processamento de Alimentos-: Princípios e Prática. Artmed Editora, 2018.

FERNANDES, F. A. N.; RODRIGUES, S.; GARCIAPEREZ, J.V.; CARCEL, J. Desidratação de Maçãs (Malus Domestica L.) Utilizando Secagem a Ar Assistida por Ultra-Som. Blucher Chemical Engineering Proceedings, V. 1, N. 2, P. 4902-4909, 2015.

FERREIRA, M. N. Estudo da secagem de jabuticaba (polpa e casca) pelo método de camada de espuma. 2017. 109 f. Dissertação (Mestrado em Ciência e Tecnologia de Alimentos) - Universidade Federal de Goiás, Goiânia, 2017.

GARCIA, D. M. Análise de atividade de água em alimentos armazenados no interior de granjas avícolas. Acta Scientiae Veterinariae, v. 32, n. 3, p. 251-252, 2004.

GRAÇA, M. L. B. Estudo do processo de secagem de frutos do cerrado em secador de bandejas com circulação forçada de ar. 2015. Monografia (Graduação em Química Tecnológica). Universidade Federal de Brasilia. Distrito Federal, 2015.

HENZ, G. P.; COSTA, C. S. R.; CARVALHO, S.; BANCI, C. A. Como cultivar pimentão. Caderno Técnico - Cultivar Hortaliças e Frutas, n. 42, 2007, 6.p.

JAY, J.M., LOESSNER, M.J. AND GOLDEN, D.A. Modern Food Microbiology. 7th Edition, Springer Science and Business Media, Inc, 63-90, 101-125. New York, 2005.

LEMOS, O. L.; REBOUÇAS, T. N. H.; SAO JOSÉ, A. R.; VILA, M. T. R.; SILVA, K. S. Utilização de biofilme comestível na conservação de pimentão'Magali R'em duas condições de armazenamento. Bragantia, v. 66, n. 4, p. 693-699, 2007.

LEWIS, W. K. The rate of drying of solid materials. Journal of Industry and Engineering Chemistry, v. 5, p. 427-432, 1921.

MADAMBA, P. S.; DRISCOLL, R. H.; BUCKLE, K. A. Thin-layer drying characteristcs of garlic slices. Journal of Food Engineering, v.29, p.75-97, 1996.

MAROTO, J. V.; SORIA, C. B. Cultivos hortícolas al aire libre. Serie Agricultura, 788 p., v. 13, 2017.
MARTINAZZO, A. P.; CÔRREA, P. C.; RESENDE, O.; MELO, E. E. Análise e descrição matemática da cinética de secagem de folhas de capim-limão. Revista Brasileira de Engenharia Agrícola e Ambiental. Campina Grande-PB, v.11, n.3, p.301-306, 2007.

MORAES FILHO, F. C. De; OLIVEIRA, E. L.; ARAUJO N. E. M. M.; OLIVEIRA; CORREIA, R. T. P. Secagem Convectiva da Acerola (Malphigia emarginata Dc.): Aplicação de Modelos Semiteóricos. Holos, v. 1, p. 86-95, 2014.

MORGADO, C. M. A.; DURIGAN, J. F.; SANCHES, J.; GALATI, V. C.; OGASSAVARA, F. O. Conservação pós-colheita de frutos de pimentão sob diferentes condições de armazenamento e filmes. Horticultura Brasileira, p. 170-174, 2008.

RAGASSI, C. F.; MELO, R.A. de C. Recomendações para manejo da compactação do solo no contexto da produção integrada do pimentão no Distrito Federal. Embrapa Hortaliças-Comunicado Técnico (INFOTECA-E), 2017.

SANTOS, A. E.; CANUTTO, M. F. C. S.; VIEIRA SEGUNDO, J. E.D.; ALMEIDA, K. D. Modelagem matemática para a descrição da cinética de secagem do fruto da palma (Opuntia fícus indica). Revista Verde de Agroecologia e Desenvolvimento Sustentável, v. 11, n. 1, p. 01-06, 2016.

SILVA, A. G. P.; FARIA, L. J. G.; COSTA, C. M. L. Otimização do Processo de Secagem dos Frutos do Açaí (Euterpe oleracea Mart.) em Secador de Leito Fixo. Blucher Chemical Engineering Proceedings, v. 1, n. 2, p. 5743-5750, 2015.

SILVA, C. S. A. Desidratação de molho de tomate caseiro pelo método Foam Mat., 2015. Monografia (Tecnologia em Alimentos) - Instituto Federal de Educação, Ciência e Tecnologia do Rio Grande do Norte. Currais Novos, 2015.

SOUSA, E. L.; SILVA, D. S.; PEREIRA, A. L. F. P. Estudo do Processo de Secagem Convectiva de Melão. In CONGRESSO LATINO-AMERICANO, 9., E CONGRESSO BRASILEIRO DE HIGIENISTAS DE ALIMENTOS, 15., 2019, Maceió. Anais... Maceió: Colégio Brasileiro de Médicos Veterinários Higienistas de Alimentos (CBMVHA), 2019. p. 33163320.

OLIVEIRA, M. T. R.; BERBERT, P. A.; MARTINAZZO, A. P. Avaliação de modelos matemáticos na descrição das curvas de secagem por convecção de Pectis brevipedunculata (Gardner) Sch. Bip. Assessment of mathematical models for the 
description of Pectis brevipedunculata (Gardner) Sch. Bip. convective drying curves. Revista Brasileira de Plantas Medicinais, v. 15, n. 1, p. 1-12, 2013.
TSUDA, J.; CARVALHO, A.; COSTA, A.; FREIRE, F. Análise Da Secagem Convectiva De Fatias De Berinjela. Blucher Chemical Engineering Proceedings, v. 1, n. 1, p. 278-283, 2014. 